\title{
Prediction of Physiological Responses and Performance at Altitude Using the 6-Minute Walk Test in Normoxia and Hypoxia
}

\author{
Oliver R. Gibson, MSc; Alan J. Richardson, PhD; Mark Hayes, PhD; Ben Duncan, BSc; Neil S. Maxwell, PhD \\ From the Centre for Sport and Exercise Science and Medicine (SESAME), Welkin Human Performance Laboratories, University of Brighton, UK.
}

\begin{abstract}
Objective.-The 6-minute walk test (6MWT) is a reliable and valid tool for determining an individual's functional capacity, and has been used to predict summit success. The primary aim of the study was to evaluate whether a 6MWT in normobaric hypoxia could predict physiological responses and exercise performance at altitude. The secondary aim was to determine construct validity of the 6MWT for monitoring acclimatization to $3400 \mathrm{~m}$ (Cuzco, Peru).

Methods.-Twenty-nine participants performed six 6MWTs in four conditions: normoxic outdoor (NO), normoxic treadmill (NT), and hypoxic treadmill (HT) were each performed once; and hypoxic outdoor (HO) was performed three times, at 42 hours (HO1), 138 hours (HO2), and 210 hours (HO3) after arrival at Cuzco.

Results.-One-way analysis of variance revealed no difference $(P>.05)$ between NO and HO1 for 6MWT distance. HT and HO protocols were comparable for the measurement of delta heart rate (HR) and post-test peripheral oxygen saturation $\left(\% \mathrm{SpO}_{2} ; P>.05\right)$. Acclimatization was evidenced by reductions $(P<.05)$ in resting HR and respiratory rate $(\mathrm{RR})$ between $\mathrm{HO} 1, \mathrm{HO} 2$, and $\mathrm{HO} 3$, and preservation of $\mathrm{SpO}_{2}$ between $\mathrm{HO} 1$ and $\mathrm{HO} 2$. Postexercise HR and RR were not different $(P>.05)$ with acclimatization. The duration to ascend to $4215 \mathrm{~m}$ on a trek was moderately correlated $(P<.05)$ to HR during the trek and the 6MWT distance during HT; no other physiological markers predicted performance.

Conclusions.-The 6MWT is a simple, time-efficient tool for predicting physiological responses to simulated and actual altitude, which are comparable. The 6MWT is effective at monitoring elements of acclimatization to moderate altitude.
\end{abstract}

Key words: 6-minute walk test, altitude sickness, hypoxia, mountaineering

\section{Introduction}

Acute altitude exposure can lead to acute mountain sickness (AMS), with $48 \%$ to $51 \%$ of travelers to Cuzco, Peru, reporting symptoms. ${ }^{1,2}$ Prolonged exposures at elevations greater than $1500 \mathrm{~m}$ are sufficient to induce AMS, which is on the high altitude illness spectrum with potential to progress to high altitude pulmonary edema and high altitude cerebral edema if untreated. ${ }^{3,4}$ Physical exertion increases AMS, with increased physiological strain placed on cardiac, pulmonary, vascular, and muscular systems. The development of a simple and

Corresponding author: Oliver R. Gibson, MSc, School of Sport and Service Management, Welkin Laboratories, University of Brighton, Eastbourne, BN20 7SR United Kingdom (e-mail: o.r.gibson@ brighton. ac.uk). efficient test for monitoring changes in physiological responses and symptoms of AMS before travel and at altitude $^{5}$ would be beneficial in aiding the identification of individuals at risk of altitude illness.

The 6-minute walk test (6MWT) has been widely used in clinical and research settings to determine and monitor exercise capacity. Performance can be linked to rates of ascent, and physiological responses to exercise at altitude can be determined. ${ }^{5,6}$ Previously, a peripheral oxygen saturation $\left(\mathrm{SpO}_{2}\right)$ greater than $75 \%$ after a $6 \mathrm{MWT}$ at a 4365-m base camp was demonstrated to be a useful screening test for predicting the outcome of successfully reaching the summit of Aconcagua (6962 m). ${ }^{5}$ More recently, however, Daniels ${ }^{6}$ concluded that $\mathrm{Spo}_{2}$ and 6MWT performance were unlikely to be effective in predicting summit success on Kilimanjaro $(5895 \mathrm{~m})$. If the data demonstrate a good relationship between 
performances in the 6MWT and physiological responses to altitude and summit success, then the 6MWT may be a useful test.

The primary aim of the study was to evaluate whether a 6MWT in normobaric hypoxia could predict physiological responses and exercise performance at altitude. Second, we aimed to determine construct validity of the 6MWT for monitoring acclimatization to $3400 \mathrm{~m}$ (Cuzco, Peru).

\section{Method}

Twenty-nine (14 female) healthy participants (age, 22.2 \pm 5.4 years, with no prior history of AMS and no exposure to simulated or actual altitude for 8 weeks before commencement of study; see Table 1 for descriptive data on day of test) volunteered to participate in an 18-day project in Eastbourne, UK, and Cuzco, Peru. After a full description of experimental procedures, the protocol was approved by the University of Brighton ethics committee. All participants completed medical questionnaires and provided written informed consent following the principles outlined by the Declaration of Helsinki of 1975, as revised in 2008.

\section{PRELIMINARY TESTING}

Anthropometric data were collected for height $(\mathrm{cm}$; Detecto Physicians Scales; Cranlea \& Co, Birmingham, UK), body mass (kg; Adam GFK 150, Adam Equipment Co Ltd, Danbury, CT), and percentage body fat obtained after multifrequency bioelectrical impedance analysis (Xitron 4000, San Diego, CA) after 20 minutes of supine rest. Hydration status was confirmed in accordance with established guidelines to reduce the potential for fluiddependent changes in AMS.,

\section{6-MINUTE WALK TESTING}

Each participant completed testing on six occasions at which a first familiarization and then experimental 6MWT were performed to permit habituation to the method and environment, and to ensure reliability on each day. ${ }^{9}$ Ten minutes of seated rest was provided between familiarization and experimental trials. Only data from the experimental 6MWT were analyzed. Participants performed a normoxic treadmill (NT) test, normoxic outdoor (NO) test, and a hypoxic treadmill test (HT) within a 7-day period (all at sea level, $760 \mathrm{~mm} \mathrm{Hg}$ ) separated with 24 hours of rest. After arrival in Cuzco, Peru (altitude approximately $3400 \mathrm{~m}, 460 \mathrm{~mm} \mathrm{Hg}$ ), 3 additional hypoxic outdoor tests were performed at 42 (HO1), 138 (HO2), and 210 (HO3) hours. Participants performed all 6MWTs in identical athletic attire between

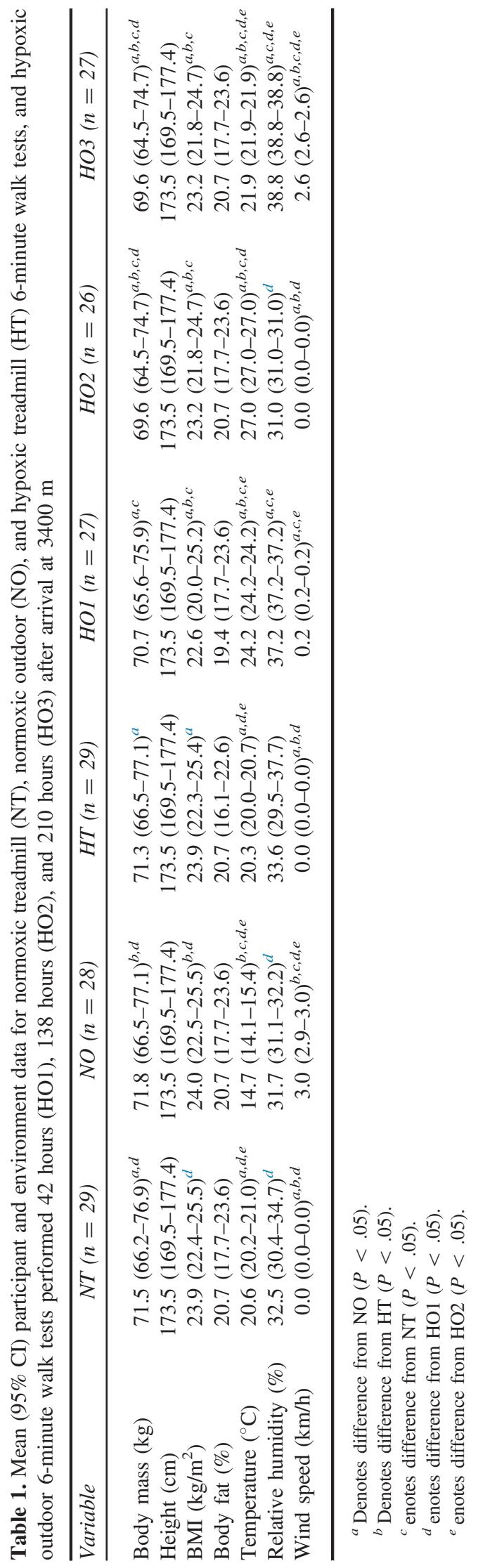


0900 and 1200 hours; data were collected during the month of April. Environmental conditions are presented in Table 1. Standardized instructions were provided before each test as follows: "Walk as far as possible in 6 minutes without running or jogging," and every 60second duration was communicated until the final 60 seconds, when 30 seconds remaining was also communicated. During the treadmill familiarization trials, participants were asked to self-select a treadmill start speed they could maintain for 6 minutes. Participants began each experimental trial at $50 \%$ of their self-selected start speed, which was doubled within 5 seconds and then obscured from the view of the participant. Participants signaled to increase speed, decrease speed, or stop the treadmill as needed.

The NT 6MWT was performed on a treadmill (ELG 2, Woodway Gmbh, Weil am Rhein, Germany) in temperate $\left(20^{\circ} \mathrm{C}, 40 \%\right.$ relative humidity) laboratory conditions $\left(\mathrm{FIO}_{2}=0.2093\right)$. The HT 6MWT was performed on a treadmill (PPS 55sport, Woodway) in a purpose-built hypoxic chamber (The Altitude Centre, London, UK) set at $\mathrm{FIO}_{2}=0.137$; approximately $3400 \mathrm{~m}$ and $20^{\circ} \mathrm{C}$ to simulate the field-testing location (Cuzco, Peru). The NO 6MWT was performed outside on level concrete tennis courts, and the HO 6MWT was performed in Cuzco, Peru, on a stadium tartan athletic track; for each trial a measured distance of $40 \mathrm{~m}$, with 5-m intervals, was demarcated. Temperature and humidity were monitored by a portable heat stress meter (HT30, Extech Instruments Corp, Nashua, $\mathrm{NH}$ ) and wind speed via a wind anemometer (EA3010, Technoline LLC, Clifton, NJ) for both outdoor trials (Table 1). Before all trials, a Lake Louise Score (LLS) was self-reported as an indicator of AMS. During all trials, heart rate (HR) and $\mathrm{SpO}_{2}$ were measured before and immediately after the test in a seated position using a pulse oximeter (Nonin 2500, Nonin Medical Inc, Plymouth, MN) affixed to the right index finger. Respiratory rate (RR) was counted over a 30-second period commencing on sitting.

In addition to 6MWTs in Cuzco, participants also performed a 4-day trek carrying day packs and dressed in typical trekking attire commencing 24 hours after HO3. Before departure LLS was recorded to determine AMS symptoms. The duration taken to reach the summit (Dead Woman's Pass, Peru, 4215 m, 48 hours after HO3) from the camp (3459 m) was recorded for each participant with $\mathrm{HR}$ and $\mathrm{Spo}_{2}$ taken immediately on reaching the pass.

\section{STATISTICAL ANALYSIS}

All statistical calculations were performed using PASW software version 20.0 (SPSS, Chicago, IL). All outcome variables were assessed for normality of distribution and sphericity before further analysis and met these criteria in all instances unless otherwise stated. Data are reported as mean $(95 \% \mathrm{CI})$, with two-tailed significance accepted at $P<.05$. One-way analysis of variance with repeated measures was used to compare NT, NO, HT, HO1, HO2, and $\mathrm{HO} 3$ data. Bonferroni pairwise comparisons compared results between trials to determine the differences between tests. Pearson's correlations $(r)$ were used to examine the relationships between dependent variables in HT and HO1, and for comparisons of the trek data with $\mathrm{HT}, \mathrm{HO} 1$, and $\mathrm{HO} 3$.

\section{Results}

Twenty-three of the 29 participants completed all tests. One participant was unable to complete the NO trial because of an acute musculoskeletal injury. Three people were excluded from the $\mathrm{HO} 2$ dataset as a result of diarrhea and vomiting $(\mathrm{n}=2)$ and severe AMS symptoms $(n=1)$. Two different individuals were excluded from the $\mathrm{HO} 3$ data set as a result of diarrhea and vomiting. Each of the ill participants' data were within the mean \pm SD for six minute walk distance (6MWD) and posttrial physiological responses during $\mathrm{HO} 1$ and are therefore considered unremarkable.

\section{HYPOXIC TREADMILL 6-MINUTE WALK TEST VS HYPOXIC OUTDOOR 6-MINUTE WALK TEST}

A difference $(P<.05)$ was observed between HT and $\mathrm{HO1}$ (Table 2) for 6MWD, LLS, HR $\mathrm{Hre}, \mathrm{HR}_{\mathrm{post}}, \mathrm{SpO}_{2 \mathrm{pre}}$, change in $(\Delta) \mathrm{Spo}_{2}, \mathrm{RR}_{\text {pre }}, \mathrm{RR}_{\text {post }}$, and $\Delta \mathrm{RR}$. No correlation $(P>.05)$ was observed for $\mathrm{Spo}_{2 \text { pre }}$, $\Delta \mathrm{SpO}_{2}, \mathrm{RR}_{\text {pre }}, \mathrm{RR}_{\text {post }}$, or $\Delta \mathrm{RR}$ (Figure). No difference $(P>.05)$ was observed between HT and HO1 for $\Delta \mathrm{HR}$ and $\mathrm{SpO}_{2 \text { post }}$ with significant $(P<.05)$ relationships observed between HT and HO1 trials for $\mathrm{HR}_{\text {pre }}(r=$ $0.753), \mathrm{HR}_{\text {post }}(r=0.721), \Delta \mathrm{HR}(r=0.538), \mathrm{Spo}_{2 \text { post }}$ $(r=0.545)$, and 6MWD $(r=0.614)$.

NORMOXIC TREADMILL 6-MINUTE WALK TEST VS NORMOXIC OUTDOOR 6-MINUTE WALK TEST

No differences $(P>.05)$ were observed between NT and NO for 6MWD, LLS, $\mathrm{HR}_{\text {pre }}, \mathrm{HR}_{\text {post }}, \Delta \mathrm{HR}, \mathrm{Spo}_{2 \text { pre }}$, $\mathrm{SpO}_{2 \text { post}}, \Delta \mathrm{SpO}_{2}, \mathrm{RR}_{\text {pre }}, \mathrm{RR}_{\text {post }}$, and $\Delta \mathrm{RR}$ (Table 2).

\section{NORMOXIC OUTDOOR 6-MINUTE WALK TEST} VS HYPOXIC OUTDOOR 6-MINUTE WALK TEST

Differences $(P<.05)$ were observed between NO and $\mathrm{HO} 1$ for LLS, $\mathrm{HR}_{\text {pre }}, \mathrm{HR}_{\text {post }}, \Delta \mathrm{HR}, \mathrm{SpO}_{2 \text { pre }}, \mathrm{SpO}_{2 \text { post }}$, 
Table 2. Mean (95\% CI) distance covered and physiological responses to normoxic treadmill (NT), normoxic outdoor (NO), and hypoxic treadmill (HT) 6-minute walk tests, and hypoxic outdoor 6-minute walk tests performed 42 hours (HO1), 138 hours (HO2), and 210 hours (HO3) after arrival at $3400 \mathrm{~m}$

\begin{tabular}{|c|c|c|c|c|c|c|}
\hline Variable & $N T(n=29)$ & $N O(n=28)$ & $H T(n=29)$ & $\mathrm{HOl}(n=29)$ & HO2 $(n=26)$ & HO3 $(n=27)$ \\
\hline 6MWD (m) & 791 (764-817) & 788 (757-819) & $774(744-805)$ & $811(778-845)^{b}$ & $800(757-843)$ & $825(793-857)^{b, c}$ \\
\hline LLS & $0.8(0.4-1.1)$ & $0.8(0.4-1.1)$ & $0.8(0.4-1.1)$ & $2.0(1.1-2.9)^{a, b, c}$ & $1.0(0.3-1.6)$ & $1.0(0.4-1.6)$ \\
\hline \multicolumn{7}{|c|}{ HR (beats/min) } \\
\hline Pre & 83.1 (74.5-91.7) & $82.0(75.7-88.3)$ & 84.7 (79.3-90.2) & $100.5(95.8-105.2)^{a, b, c, d, e}$ & $85.1(95.8-105.2)$ & 80.5 (75.4-85.7) \\
\hline Post & $125.6(111.7-139.4)^{b}$ & $116.3(103.4-129.2)^{b}$ & $141.0(132.5-149.5)^{a, c}$ & $154.7(147.2-162.1)^{a, b, c}$ & $148.1(138.5-157.7)^{a, c}$ & $149.1(143.0-155.3)^{a}$ \\
\hline Delta & $48.2(36.9-59.5)$ & $34.3(23.9-44.8)^{b, d, e}$ & $56.3(49.4-63.1)^{a}$ & $54.2(47.9-60.5)^{a}$ & $63.0(52.4-73.6)^{a}$ & $68.6(61.0-76.3)^{a}$ \\
\hline \multicolumn{7}{|l|}{$\mathrm{SpO}_{2}(\%)$} \\
\hline Pre & $97.3(96.6-97.9)^{b, d, e}$ & $97.7(96.6-98.7)^{b, d, e}$ & $88.7(87.3-90.2)^{a, c, d, e}$ & $92.5(91.6-93.3)^{a, b, c}$ & $91.6(90.8-92.4)^{a, b, c}$ & $92.2(91.4-93.0)^{a, b, c}$ \\
\hline Post & $96.3(97.5-98.6)^{b, d, e}$ & $98.0(97.5-98.6)^{b, d, e}$ & $83.7(81.5-85.9)^{a, c}$ & $83.5(81.8-85.2)^{a, c, d}$ & $86.7(85.2-88.2)^{a, c}$ & $85.4(83.7-87.2)^{a, c}$ \\
\hline Delta & $-1.0(-1.8 \text { to }-0.1)^{b, d, e}$ & $0.4(-1.0 \text { to } 1.7)^{b, d, e}$ & $-5.0(-6.5 \text { to }-3.5)^{a, c}$ & $-9.0(-10.7 \text { to }-7.2)^{a, b, c, d}$ & $-4.9(-6.2 \text { to }-3.5)^{a, c}$ & $-6.7(-8.4 \text { to }-5.1)^{a, c}$ \\
\hline \multicolumn{7}{|c|}{ RR (breaths/min) } \\
\hline Pre & $7.6(6.9-8.4)$ & $7.2(6.2-8.2)$ & $7.7(6.8-8.5)$ & $8.8(7.7-9.9)^{a, b, c, d, e}$ & $7.6(6.9-8.3)$ & $7.1(6.3-7.9)$ \\
\hline Post & $11.6(10.6-12.6)^{d, e}$ & $10.6(9.1-12.1)^{d, e}$ & $11.6(10.4-12.8)^{d, e}$ & $18.7(16.6-20.8)^{a, b, c}$ & $16.8(14.3-19.3)^{a, b, c}$ & $18.9(16.8-21.0)^{a, b, c}$ \\
\hline Delta & $4.0(3.0-5.0)^{d, e}$ & $3.4(2.4-4.4)^{d, e}$ & $3.9(2.9-4.9)^{d, e}$ & $9.9(7.8-12.0)^{a, b, c}$ & $9.3(6.9-11.6)^{a, b, c}$ & $11.7(9.8-13.7)^{a, b, c}$ \\
\hline
\end{tabular}

\footnotetext{
${ }^{a}$ Denotes difference from NO $(P<.05)$

${ }^{b}$ Denotes difference from HT $(P<.05)$.

${ }^{c}$ Denotes difference from NT $(P<.05)$.

${ }^{d}$ Denotes difference from $\mathrm{HO} 2(P<.05)$.

${ }^{e}$ Denotes difference from HO3 $(P<.05)$.
} 

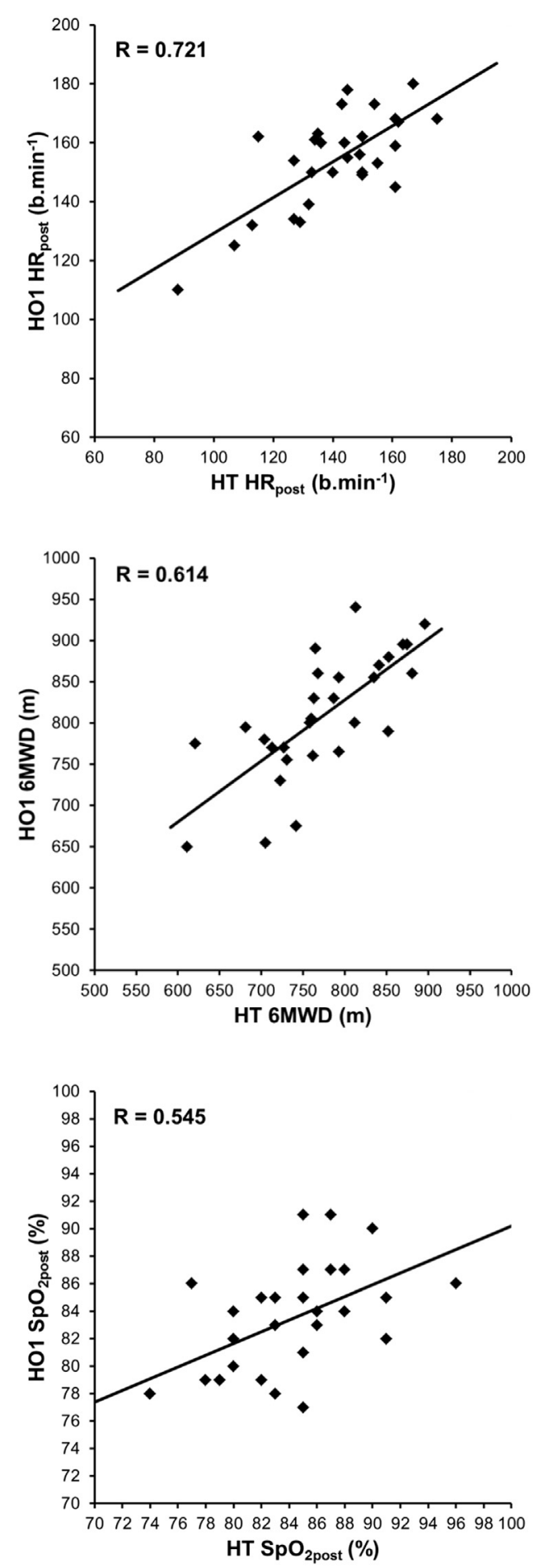

Figure. Relationships between post 6-minute walk test (6MWT) heart rate (HR), total 6-minute walk duration (6MWD), and peripheral oxygen saturation $\left(\mathrm{Spo}_{2}\right)$ in normobaric hypoxic treadmill test (HT) and the hypobaric hypoxic outdoor test performed 42 hours (HO1) after arrival at $3400 \mathrm{~m}$ for all participants.
$\Delta \mathrm{SpO}_{2}, \mathrm{RRpre}, \mathrm{RR}_{\text {post }}$, and $\Delta \mathrm{RR}$. No difference $(P>$ $.05)$ was observed between NO and HO1 for 6MWD.

\section{HYPOBARIC HYPOXIC COMPARISONS-THE EFFECT OF ACCLIMATIZATION}

Performance and physiological variables $6 \mathrm{MWD}$, LLS, $\mathrm{HR}_{\text {pre }}, \mathrm{HR}_{\text {post }}, \Delta \mathrm{HR}, \mathrm{Spo}_{2 \text { pre, }} \mathrm{Spo}_{2 \text { post }}, \Delta \mathrm{Spo}_{2}, \mathrm{RR}_{\text {pre }}$, $\mathrm{RR}_{\text {post }}$, and $\triangle \mathrm{RR}$ reported differences $(P<.05)$ between $\mathrm{NO}, \mathrm{HT}, \mathrm{HO} 1, \mathrm{HO} 2$, and $\mathrm{HO} 3$; post hoc analysis is detailed in Table 2.

\section{TREK DATA}

Time taken to complete the ascent to Dead Woman's Pass (157 minutes; CI, 144-171) was weakly correlated with HR $(\mathrm{r}=0.420 ; P<.05)$ and the HT 6MWD $(r=$ 0.407; $P<.05$ ). Additionally, $\mathrm{SpO}_{2}$ after the ascent (78.2\%; CI, 76.4-79.9) was weakly correlated with the $\Delta$ RR during HO3 $(r=0.391 ; P<.05)$; no relationships were observed for the LLS $(0.9$; CI, 0.4-1.31) before the ascent.

\section{Discussion}

Our data demonstrate that $\Delta \mathrm{HR}$ and posttest $\% \mathrm{Spo}_{2}$ were correlated between hypoxic treadmill and hypoxic outdoor 6MWT protocols. Other physiological or performance markers are not correlated. In accordance with our primary aim, these variables are therefore appropriate for use to determine physiological responses to simulated altitude before travel and on immediate arrival at altitude. AMS symptoms were not clinically relevant or related to performance of any 6MWT.

In comparison with the normoxic outdoor baseline trial, no differences were observed in the distance walked during the hypoxic outdoor trials. Acclimatization was evidenced by reductions in resting $\mathrm{HR}$ and RR, although postexercise $\mathrm{HR}, \mathrm{SpO}_{2}$, and $\mathrm{RR}$ and the change in variables were not different with acclimatization. This might suggest that the physiological challenge of exercising in hypoxia in comparison with normoxia is too great to overcome, or the sensitivity or construct validity of the test is inadequate. Our hypoxic outdoor trial data suggest participants were able to walk a greater distance and, consequently, elicited more favorable physiological responses (increased HR, decreased RR, and preserved $\mathrm{SpO}_{2}$ after the test) at $3400 \mathrm{~m}$ in Cuzco, in comparison with a similar study performed at $4365 \mathrm{~m}^{5}$ This is unsurprising because of the differences in $\mathrm{Po}_{2}$ at these locations. 
The duration taken to reach the peak ascent (Dead Woman's Pass), a field-based performance indicator, was moderately related to the 6MWD in the HT trial, suggesting that the exercise capacity of an individual is important in governing the speed of ascent. The relationship between ascent time and the HR during the ascent also provides a useful performance indicator. The lack of relationship between the duration taken to ascend and any other physiological or AMS marker suggests that $\mathrm{SpO}_{2}$ and 6MWD are unlikely to be effective tools for predicting successful ascent. ${ }^{6}$ It has been suggested that with AMS symptoms likely among climbers, psychological factors would more likely dictate peak or summit success. ${ }^{6}$ The success of reaching a pass or summit of a peak is dependent on a number of variables, including acclimatization, psychological factors, weather, impaired sleep, inflammation, fluid shifts, and hematopoiesis; thus success cannot be fully elucidated with vital signs in a small group alone.

\section{LIMITATIONS}

The severity of AMS symptoms peak between days 1 and 3 of arrival at altitude, ${ }^{10}$ and acclimatization tends to occur during the first week. Therefore, the identification of no differences between $\mathrm{HO} 2$ and $\mathrm{HO} 3$ is unsurprising, and future studies should implement earlier and morefrequent analysis. The authors acknowledge that differences in participant approach to outdoor and treadmill walking, eg, pacing, may have affected findings. Further, deceleration and acceleration associated with turning during outdoor walking may have reduced strain. Finally, the rate of ascent and physiological data may have been affected by pacing, eg, additional rest breaks or faster walking at the end of the ascent, continuous monitoring of $\mathrm{HR}$ and $\mathrm{O}_{2}$, and walking velocity via GPS would be more beneficial.

\section{Conclusions}

The 6MWT is a useful and simple tool for determining performance and physiological responses to self-paced exercise in hypoxia that can be administered using a treadmill and over level ground outdoors. The implementation of the 6MWT warrants further investigation as a means for predicting for responses to acute exposures and altitude acclimatization, via the postexercise changes in physiological responses to, but not performance during, the 6MWT. Preliminary data suggest the 6MWT may be useful to determine acclimatization to altitude as attenuation of physiological responses (change in $\mathrm{HR}$ and posttest $\mathrm{SpO}_{2}$ ) from baseline tests.

\section{Acknowledgements}

The authors wish to acknowledge Cortex-Medical, MKK Sports, Mad Designs, Para-Monte, and Cranlea for their support with sponsoring the expedition to Cuzco, Peru, and equipment used.

\section{References}

1. Salazar H, Swanson J, Mozo K, White AC Jr, Cabada MM. Acute mountain sickness impact among travelers to Cusco, Peru. J Travel Med. 2012;19:220-225.

2. Merritt AL, Camerlengo A, Meyer C, Mull JD. Mountain sickness knowledge among foreign travelers in Cuzco, Peru. Wilderness Environ Med. 2007:18:26-29.

3. West JB, American College of Physicians; American Physiological Society. The physiologic basis of highaltitude diseases. Ann Intern Med. 2004;141:789-800.

4. Luks AM, McIntosh SE, Grissom CK, et al. Wilderness Medical Society consensus guidelines for the prevention and treatment of acute altitude illness. Wilderness Environ Med. 2010;21:146-155.

5. Lazio MP, Van Roo JD, Pesce C, Malik S, Courtney DM. Postexercise peripheral oxygen saturation after completion of the 6-minute walk test predicts successfully reaching the summit of Aconcagua. Wilderness Environ Med. 2010;21:309-317.

6. Daniels R. Pulse oximetry after 6-minute walk test and summit success on Kilimanjaro. Wilderness Environ Med. 2012;23:95-96.

7. Nerín MA, Palop J, Montaño JA, Morandeira JR, Vázquez M. Acute mountain sickness: influence of fluid intake. Wilderness Environ Med. 2006;17:215-220.

8. Richardson A, Watt P, Maxwell N. Hydration and the physiological responses to acute normobaric hypoxia. Wilderness Environ Med. 2009;20:212-220.

9. Pankoff BA, Overend TJ, Lucy SD, White KP. Reliability of the six-minute walk test in people with fibromyalgia. Arthritis Care Res. 2000;13:291-295.

10. West JB, Schoene RB, Luks AM, Milledge JS. High Altitude Medicine and Physiology. 5th Edition. CRC Press; 2012. 